Strohmeier, Daniel; Kowalski, Matthieu; Haueisen, Jens; Hämäläinen, Matti S.;

Gramford, Alexandre:

MEG/EEG single trial source estimates based on inter-trial priors in the timefrequency domain for source connectivity analysis

Zuerst erschienen in: Biomedical Engineering = Biomedizinische Technik. - Berlin [u.a.] : de Gruyter. - 57 (2012), Suppl. 1, Track-F, p. 391.

Erstveröffentlichung: $\quad$ 2012-08-30

ISSN (online): $\quad$ 1862-278X

ISSN (print): $\quad$ 0013-5585

DOI: $\quad 10.1515 / \mathrm{bmt}-2012-4461$

[Zuletzt gesehen: 2019-08-12]

„Im Rahmen der hochschulweiten Open-Access-Strategie für die Zweitveröffentlichung identifiziert durch die Universitätsbibliothek IImenau. “

"Within the academic Open Access Strategy identified for deposition by Ilmenau University Library."

„Dieser Beitrag ist mit Zustimmung des Rechteinhabers aufgrund einer (DFGgeförderten) Allianz- bzw. Nationallizenz frei zugänglich."

"This publication is with permission of the rights owner freely accessible due to an Alliance licence and a national licence (funded by the DFG, German

DFG

Research Foundation) respectively." 


\section{MEG/EEG single trial source estimates based on inter-trial priors in the time-frequency domain for source connectivity analysis}

Strohmeier D., Institute of Biomedical Engineering and Informatics, Ilmenau University of Technology, Ilmenau, Germany, daniel.strohmeier@tu-ilmenau.de

Kowalski M., Laboratoire des Signaux et Systèmes (CNRS-SUPELEC-Univ. Paris-Sud)

SUPELEC, Plateau de Moulon, Gif-sur-Yvette cedex, France, matthieu.kowalski@1ss.supelec.fr

Haueisen J., Institute of Biomedical Engineering and Informatics, Ilmenau University of Technology, Ilmenau, Germany, jens.haueisen@tu-ilmenau.de

Hämäläinen M., Martinos Center for Biomedical Imaging, Department of Radiology, Massachusetts General Hospital, Boston, MA,msh@nmr.mgh.harvard.edu

Gramfort A., Parietal Project Team, INRIA Saclay Ile-de-France, CEA - NeuroSpin, Gif-sur-Yvette cedex, France, alexandre.gramfort@inria.fr

\section{Introduction}

Analysing functional connectivity of neuronal sources based on MEG/EEG source estimates is a non-invasive approach to examine the brain functioning. In order to apply functional connectivity measures such as coherency and phase locking value (PLV) to evoked responses, which are highly non-stationary over time, the source activations and the respective complex-valued time-frequency distributions have to be estimated on a single-trial basis. However, due to the low signal-to-noise ratio of unaveraged data, the applicability of present source reconstruction methods to the study of single-trial MEG/EEG data is limited.

\section{Methods}

We apply the multi-trial time-frequency mixed-norm estimate (MT-TF-MxNE) for reconstructing single-trial source estimates, which is based on a composite multidimensional mixed norm prior in the time-frequency domain combining a sparse intra-trial prior and a smooth inter-trial prior. By thresholding the Gabor transform of the source signals, the intra-trial prior promotes spatial sparsity and temporal smoothness of the neuronal activity per trial. The inter-trial prior retains consistency of the respective sparsity structure in the time-frequency domain to impose stationarity over trials. Amplitudes and phases of the active time-frequency coefficients are allowed to vary in order to account for trial-to-trial variations. Thus, the MT-TF-MxNE computes single-trial estimates while regarding multiple trials simultaneously to increase the information content. The resulting sparse regression problem is a convex optimization problem, which is solved using the fast iterative shrinkage-thresholding algorithm. Subsequently, for analysing source connectivity, coherency, coherence and imaginary coherence, and the PLV can be computed using the reconstructed single-trial timefrequency distributions.

\section{Results}

The MT-TF-MxNE is applied to simulated MEG/EEG data showing its ability to reconstruct location, activation and time-frequency distribution of evoked neuronal activity for functional source connectivity analysis.

\section{Conclusion}

The results show that the MT-TF-MxNE is a promising source reconstruction approach for analysing evoked MEG/EEG data. Applying the MT-TF-MxNE to real MEG/EEG data is part of the future work. 